\title{
Applied Systems Biology—embracing molecular imaging for systemic medicine
}

\author{
Marcus Hacker ${ }^{1} \cdot$ Rodney J. Hicks ${ }^{2} \cdot$ Thomas Beyer $^{3}$ \\ Published online: 7 April 2020 \\ (C) Springer-Verlag GmbH Germany, part of Springer Nature 2020
}

“... our empirical knowledge is a compound of that which we receive through impressions, and that which the faculty of cognition supplies from itself ..."

[Immanuel Kant, 1724-1804]

\section{Dynamic whole-body positron emission tomography and Applied Systems Biology}

Our understanding of complex diseases remains rudimentary and limits our ability to make rational and effective treatment choices. Although molecular technologies have dramatically improved our insights into pathological mechanisms of disease, molecular biology approaches fall short of deconvolving the complex signaling pathways involved in disease within living organisms and, while operating at the microscopic scale, have limited capability for interrogating heterogeneity in space and over time. On the other hand, diagnostic imaging methods, particularly including positron emission tomography (PET), support non-invasive probing of metabolic and signaling pathways on a whole-body scale, but are constrained by the physical limits of spatial and contrast resolution of current instrumentation. Recent advances in extended fieldof-view PET/CT systems will, however, expand the spatiotemporal performance of molecular imaging through highly-

This article is part of the Topical Collection on Editorial

Marcus Hacker

marcus.hacker@meduniwien.ac.at

1 Department of Biomedical Imaging and Image-guided Therapy, Division of Nuclear Medicine, Medical University Vienna, Währinger Gürtel 18-20, 1090 Vienna, Austria

2 The Sir Peter MacCallum Department of Oncology, The University of Melbourne, Parkville, VIC, Australia

3 QIMP Team, Center for Medical Physics and Biomedical Engineering, Medical University Vienna, Lazarettgasse 14, 1090 Vienna, Austria sensitive, dynamic, whole-body (DWB) imaging. We contend that the combination of DWB-PET with targeted use of established molecular biology approaches holds the promise of deepening our understanding of pathological mechanisms of disease and, thereby, guide treatment decisions more effectively. Such "Applied Systems Biology" approach is likely to advance our understanding of complex interactions during pathophysiological processes and help personalize therapies.

\section{Precision medicine}

Knowledge about biological systems has been immeasurably advanced by molecular biology, which is founded on a thorough understanding of cellular and biological interactions underpinned by the primary sciences of physics and chemistry [1]. This reductionistic perspective proved to be an extremely powerful analytical methodology that has enabled scientists to analyze many basic molecular and cellular processes down to the level of a single cell. Likewise, molecular targets and their downstream signaling pathways were successfully identified for diagnostic and therapeutic purposes, thus, defining a range of medical fields, including clinical oncology, cardiovascular medicine, neuropsychiatry, and immunology. These domains bring together new knowledge, expertise, and experience to define state-of-the-art patient management. Thanks to the application of novel molecular diagnostic approaches, including so-called "-omics" technologies, flow cytometry, and in-vivo microscopy, we have come to better understand molecular processes that cause disease and have moved from experience-based towards evidence-based care (Fig. 1).

Complementing these advances are parallel developments in our armamentarium of diagnostic imaging methods, particularly PET, which stands out as a platform for advanced, noninvasive probing of metabolic and signaling pathways [2] and is being ever more widely used for diagnostic work-up, therapeutic planning, and decision-making using a targeted approach [3]. This process, known as "personalized medicine," 


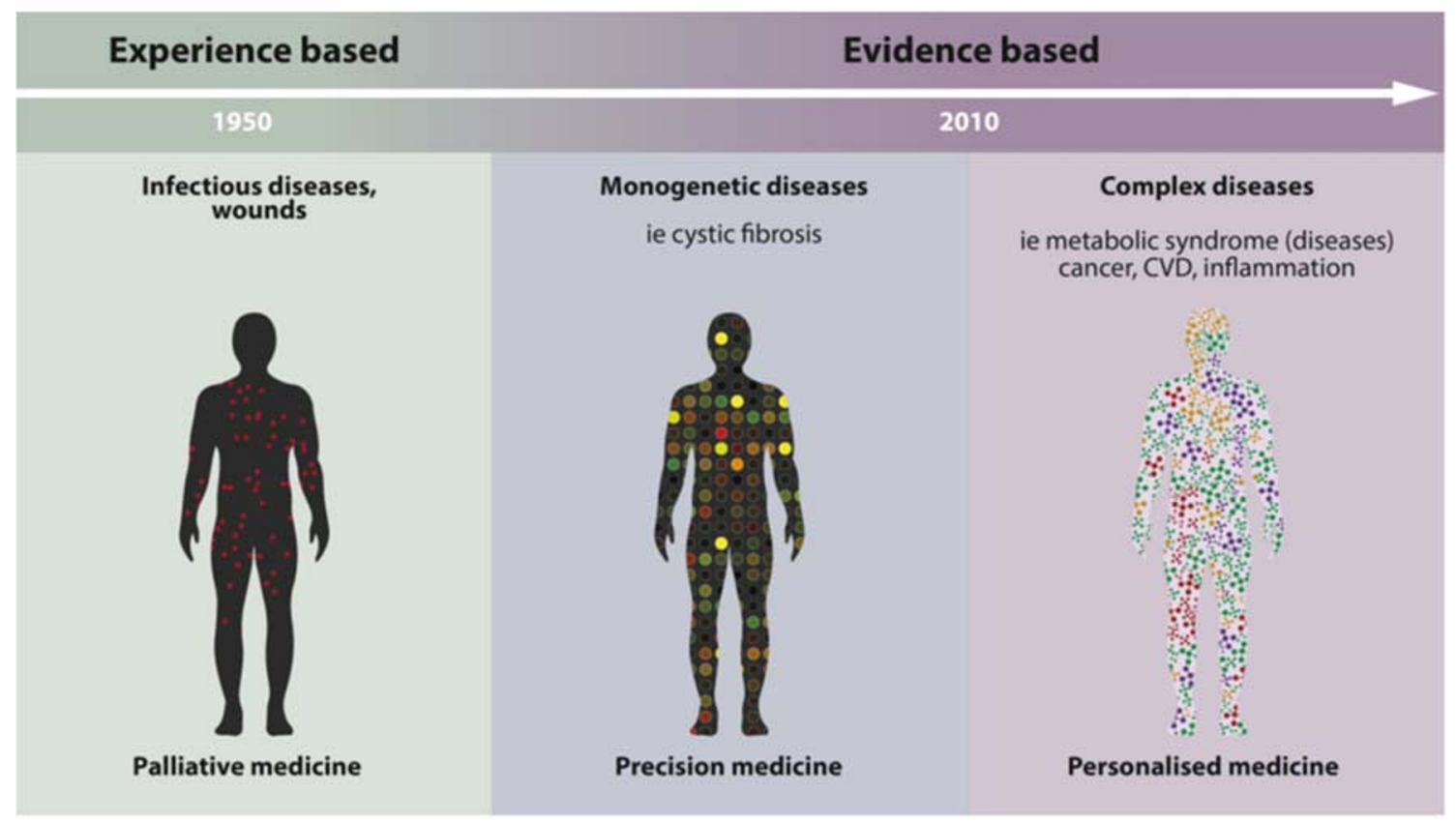

Fig. 1 Modern medicine shifts from being mainly experience-based towards evidence-based. This process is amended by the availability of

represents a societal model for customization of healthcare to individual patient's needs with positive outcomes in terms of the costs and effectiveness of therapeutic intervention, which is termed "precision medicine."

\section{Biological systems}

Virtually all pathological mechanisms involve complex interactions between molecular structures within different cell types that then impact the function of organs in living organisms and become manifest as disease. An "Applied Systems Biology" approach aims to predict the behavior of disease processes by integrating data and biomarkers rather than by interpreting the constituent parts independently. Embracing advances in "-omics" technologies, tissue and blood biomarkers, imaging methods, computer sciences, and bioinformatics, Systems Biology has become a key approach for understanding disease and thereby combatting it.

Independently, the field of developmental biology analyzes self-organization in suitable model systems. For example, the time-resolved observation of development in transparent fish or worm embryos provided revolutionary insights into biological systems, emphasizing the importance of spatio-temporal analysis [4]. Similarly, DWB-PET provides a means to visualize, quantify, and interrogate pertinent inter-organ and intrabody signaling in an extended temporal domain and beyond the organ-level imaging previously achievable on scanners with a limited field-of-view and relatively low sensitivity [5-8]. novel diagnostic methods (including -omics technologies and imaging). Figure reproduced with permission of BIOLUTION GmbH, Austria

\section{Interaction of Systems Biology with disease}

Today, the relevance of systemic factors in modulating disease development, progression, and treatment response is widely recognized. For example, the immune system plays a vital role in the outcome of oncological diseases. Here, the innate and adaptive immune systems make use of complex tissue and environmental signals that can either control or facilitate tumor growth. In turn, these responses regulate physiologic processes in multiple tissues, including the nervous system, or by impacting metabolic status [9, 10]. Perturbing the immune system therapeutically can have profound effects, not only on tumors but also on normal tissues, as evidenced by the frequent occurrence of immune-related adverse events in response to immune-checkpoint inhibitors.

Similarly, psychosocial factors can influence patterns of disease, for example the progression of atherosclerosis [11] with neural and hormonal pathways invoking stress-induced immune cell proliferation in vascular plaques [12]. Such interactions between disease processes and normal organs are bidirectional in that, for example, cytokines produced by immune cells can modulate the activity of the hypothalamus [13].

\section{Metabolic Syndrome as an exemplar of Applied Systems Biology}

The Metabolic Syndrome (MetS) provides an ideal model for analyzing complex, but disease-specific interactions. The 
MetS is defined by a cluster of entangled factors that directly increase the risk of Type 2 diabetes mellitus (T2DM), nonalcoholic fatty liver disease (NAFLD) with steatohepatitis (NASH) as more progressive variant, cardiovascular diseases, and cancer. It is phenotypically characterized by intraabdominal fat deposition and biochemically by insulin resistance. Interaction between disturbed cellular metabolism and inflammatory responses, involving a complex array of mediators, impacts development of disease in a manner that defies analysis using conventional cell biology and molecular approaches/studies, but could be augmented by whole-body molecular imaging capability provided by DWB-PET.

This has been available using preclinical imaging devices for many years. For example, serial whole-body PET imaging of the mitochondrial translocator protein $\left(\left[{ }^{18} \mathrm{~F}\right] \mathrm{TSPO}\right.$, a marker of both activated macrophages and microglia in mice, demonstrated a concomitant immune activation in the myocardium and brain after myocardial infarction [14]. However, this capability has been constrained in humans to an evaluation of changes in specific organs. For example, PET imaging, employing $\left[{ }^{18} \mathrm{~F}\right] \mathrm{FDG}$ as a surrogate marker for glucose metabolism in tissue and immune cells, demonstrated a link between amygdala activity invoked by immune responses and subsequent cardiovascular disease [15]. Similarly, PET imaging was used to demonstrate the effects of steroidal hormone-mediated signaling on brain function and behavior in psychiatric disorders, such as anxiety and depression [16]. Another example is the association of metabolic activity of the resting-state amygdala with abnormal cardiac function and perfusion in women, thus, suggesting a link between emotional stress and cardiovascular disease [17]. However, all of these studies focused on imaging the brain and not the target organ eventually impacted, the heart.

Novel, DWB-PET imaging approaches are based on innovative acquisition schemes with limited axial field-of-view PET systems [6], or recently engineered total-body PET imaging systems [5]. These concepts support absolute quantification of target expression of the organism in toto over time and, thus, yield insights into the interplay of molecular processes and signaling pathways, such as inflammation, neurohormonal activation, and metabolic processes. They also lay the grounds to deciphering biological processes that were

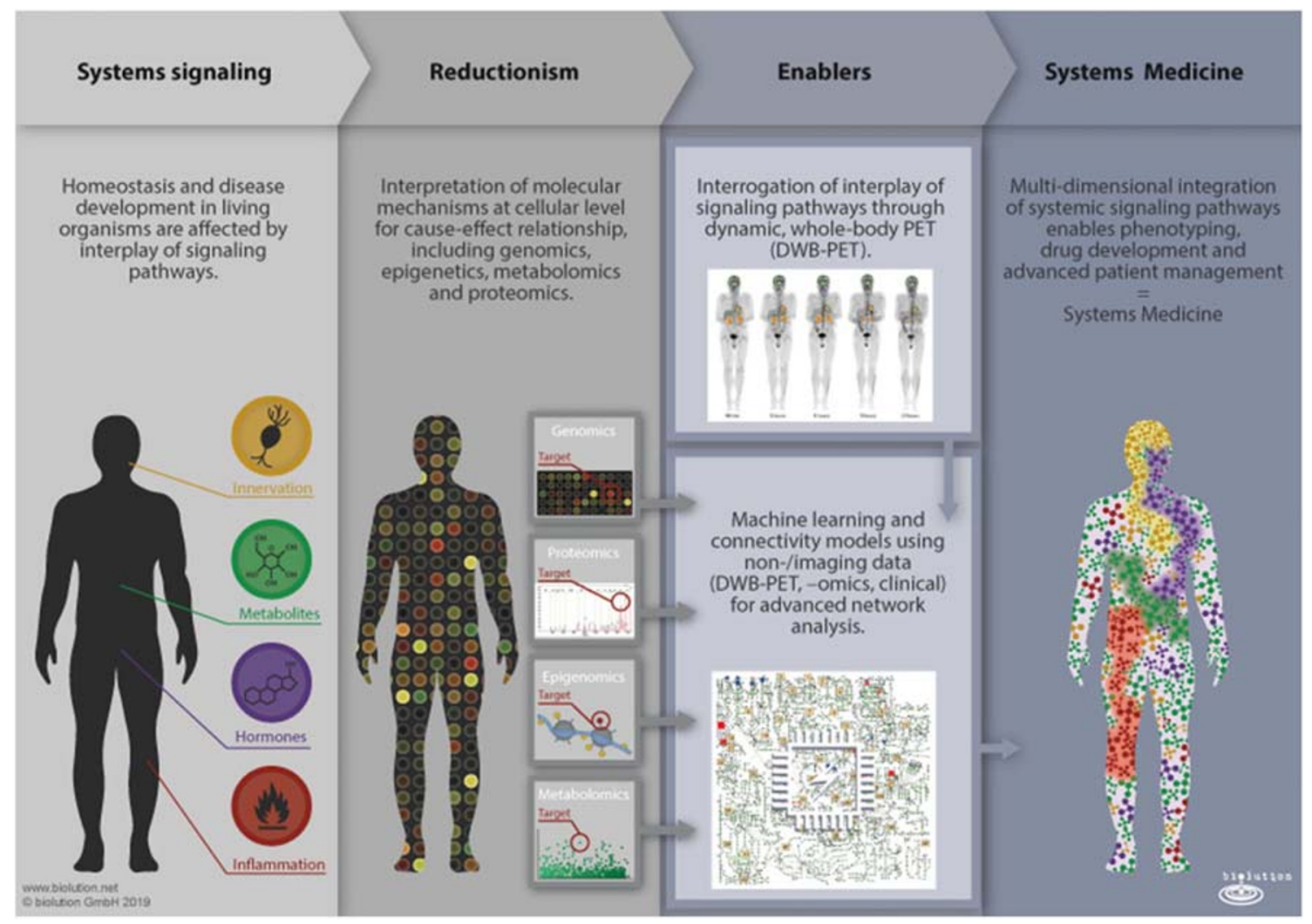

Fig. 2 Systemic signaling contributes to the genesis and development of pathological diseases, which are hypothesized to be better understood in a spatio-temporal framework of functional, molecular, biological, and genetic data. Previous biological concepts to deduce pathological mechanisms were based on reductionistic perspectives. Through the use of whole-body, molecular imaging, novel insights into the spatio-temporal domain of signaling pathways can be expected. When combined with other relevant data by employing computational methods (aka enablers), dynamic maps of inter-organ signaling can be created to advance our fundamental understanding of complex disorders in the context of an "Applied Systems Biology" approach, thus, leading to the concept of Systems Medicine. Figure reproduced with permission of BIOLUTION $\mathrm{GmbH}$, Austria 
previously assessed on a whole-body scale only through summation of biodistribution kinetics or at an organ level by regional dynamic PET imaging.

Moreover, DWB-PET can also be applied in serial studies focusing on the resolution and regression of biochemical processes after medical intervention or other stimuli. Both PET strategies are available in the preclinical scenario already, while DWB-PET in humans is only now becoming fashionable. Considering that the same radiopharmaceuticals being used for target visualization and quantification can be applied in both rodents and humans, these methods offer the option for real translational mouse-to-human and back scenarios. The heightened sensitivity of these systems can also substantially reduce the radiation exposure involved in molecular imaging and potentially allow several different probes to be employed efficiently to characterize disease processes, particularly using a combination of short and long half-life radionuclides. Furthermore, observation of phenotypic heterogeneity on a whole-body scale in chemical, physiological, or biological characteristics could allow targeted application of molecular biological techniques to understand their fundamental nature.

\section{Why do we need Applied Systems Biology?}

Precision medicine is often touted as the future application of the innovations in biological sciences or molecular imaging, but has failed to penetrate deeply into clinical practice because both technologies have tended to operate in silos. We believe that realizing better outcomes in patients will rely on optimally leveraging the capabilities of both. Through serial observations of dynamic processes and analysis of long-range signals between organs, as supported by DWB-PET in conjunction with current molecular biology and bioinformatics approaches, we can advance our understanding of complex interactions during pathophysiological processes beyond unidirectional cause-effect interpretations.

The huge amount of data generated from DWB-PET and genomic analysis will generate complex datasets mandating use of iterative artificial intelligence (AI) and machine learning (ML) processes, connectivity, and interactome network analyses (Fig. 2); all of which are areas of active engagement of many groups, yet with limited exchange and cross-silo communication. Such engagements entail significant efforts in accessing larger-scale, high-performance infrastructures that may be too costly for a single institution, thus, mandating on an additional level strong and hypothesis-driven cooperation between complementary academic and industrial partners.

We appreciate that very few of the current generation of imaging specialists have ever considered DWB-PET imaging or compartmental modeling. In fact, it took 40 years to reconcile the unique basic concept of PET, which is to non- invasively visualize and quantify metabolic and signaling pathways in living subjects [2]. The strength of integrative diagnostics built on the Applied Systems Biology concept is that, even when a perturbation in a molecular pathway does not result in clear phenotypic changes, the responsible compensatory adaptations could be mirrored in adaptations in the molecular PET datasets. The appreciation and adoption of this concept requires a truly cross-specialty and cross-domain engagement of numerous stakeholders, for the ultimate benefit of helping patients. This would be translational medicine at its best.

\section{Compliance with ethical standards}

Ethical approval Not applicable.

Informed consent Not applicable.

Conflict of interest The authors declare that they have no conflict of interest.

\section{References}

1. Crick FHC. Of molecules and men. Seattle: USA University of Washington Press; 1966.

2. Lammertsma AA. Forward to the past: the case for quantitative PET imaging. J Nucl Med. 2017;58:1019-24. https://doi.org/10.2967/ jnumed.116.188029.

3. Hacker M, Hoermann G, Kenner L. Molecular imaging and molecular diagnostics: two sides of the same coin? Eur J Nucl Med Mol Imaging. 2018;45:1645-8. https://doi.org/10.1007/s00259-0184060-x.

4. Gunsalus KC, Ge H, Schetter AJ, Goldberg DS, Han JD, Hao T, et al. Predictive models of molecular machines involved in Caenorhabditis elegans early embryogenesis. Nature. 2005;436: 861-5. https://doi.org/10.1038/nature03876.

5. Badawi RD, Shi H, Hu P, Chen S, Xu T, Price PM, et al. First human imaging studies with the EXPLORER total-body PET scanner. J Nucl Med. 2019;60:299-303. https://doi.org/10.2967/ jnumed.119.226498.

6. Karakatsanis NA, Casey ME, Lodge MA, Rahmim A, Zaidi H. Whole-body direct 4D parametric PET imaging employing nested generalized Patlak expectation-maximization reconstruction. Phys Med Biol. 2016;61:5456-85. https://doi.org/10.1088/0031-9155/ 61/15/5456.

7. Zhang X, Cherry SR, Xie Z, Shi H, Badawi RD, Qi J. Subsecond total-body imaging using ultrasensitive positron emission tomography. Proc Natl Acad Sci U S A. 2020;117:2265-7. https://doi.org/ 10.1073/pnas.1917379117.

8. Zhang X, Xie Z, Berg E, Judenhofer MS, Liu W, Xu T, et al. Totalbody dynamic reconstruction and parametric imaging on the uEXPLORER. J Nucl Med. 2020;61:285-91. https://doi.org/10. 2967/jnumed.119.230565.

9. Hanahan D, Weinberg RA. Hallmarks of cancer: the next generation. Cell. 2011;144:646-74. https://doi.org/10.1016/j.cell.2011.02. 013.

10. Rankin LC, Artis D. Beyond host defense: emerging functions of the immune system in regulating complex tissue physiology. Cell. 2018;173:554-67. https://doi.org/10.1016/j.cell.2018.03.013. 
11. Yusuf S, Hawken S, Ounpuu S, Dans T, Avezum A, Lanas F, et al. Effect of potentially modifiable risk factors associated with myocardial infarction in 52 countries (the INTERHEART study): casecontrol study. Lancet. 2004;364:937-52. https://doi.org/10.1016/ S0140-6736(04)17018-9.

12. Hassan M, Latif N, Yacoub M. Adipose tissue: friend or foe? Nat Rev Cardiol. 2012;9:689-702. https://doi.org/10.1038/nrcardio. 2012.148 .

13. Heidt T, Sager HB, Courties G, Dutta P, Iwamoto Y, Zaltsman A, et al. Chronic variable stress activates hematopoietic stem cells. Nat Med. 2014;20:754-8. https://doi.org/10.1038/nm.3589.

14. Thackeray JT, Hupe HC, Wang Y, Bankstahl JP, Berding G, Ross TL, et al. Myocardial inflammation predicts remodeling and neuroinflammation after myocardial infarction. J Am Coll Cardiol. 2018;71:263-75. https://doi.org/10.1016/j.jacc.2017.11.024

15. Tawakol A, Ishai A, Takx RA, Figueroa AL, Ali A, Kaiser Y, et al. Relation between resting amygdalar activity and cardiovascular events: a longitudinal and cohort study. Lancet. 2017;389:834-45. https://doi.org/10.1016/S0140-6736(16)31714-7.

16. Moraga-Amaro R, van Waarde A, Doorduin J, de Vries EFJ. Sex steroid hormones and brain function: PET imaging as a tool for research. J Neuroendocrinol. 2018;30. https://doi.org/10.1111/jne. 12565 .

17. Fiechter M, Roggo A, Burger IA, Bengs S, Treyer V, Becker A, et al. Association between resting amygdalar activity and abnormal cardiac function in women and men: a retrospective cohort study. Eur Heart J Cardiovasc Imaging. 2019;20:625-32. https://doi.org/ 10.1093/ehjci/jez047.

Publisher's note Springer Nature remains neutral with regard to jurisdictional claims in published maps and institutional affiliations. 